hep-th/0305137

RI-01-03

\title{
Strings in Singular Time-Dependent Backgrounds ${ }^{1}$
}

\author{
Amit Giveon $^{1}$, Eliezer Rabinovici ${ }^{2}$, Amit Sever ${ }^{3} \S$ \\ Racah Institute of Physics, The Hebrew University \\ Jerusalem, 91904, Israel.
}

\begin{abstract}
We review the construction of time-dependent backgrounds with space-like singularities. We mainly consider exact CFT backgrounds. The algebraic and geometric aspects of these backgrounds are discussed. Physical issues, results and difficulties associated with such systems are reviewed. Finally, we present some new results: a two dimensional cosmology in the presence of an Abelian gauge field described within a family of $\frac{S L(2) \times U(1)}{U(1) \times Z}$ quotient CFTs.
\end{abstract}

\section{Introduction}

The properties of supersymmetry on the worldsheet and in space-time play an important role in circumventing pitfalls in string theory. Our universe is not explicitly supersymmetric. Our universe is time-dependent. Employing the arsenal of familiar methods to treat strings in time-independent backgrounds in the cases of time-dependent ones is not straightforward. These are several of the important problems that string theorists face with some difficulty.

In this talk we discuss some issues concerning the motion of strings in time-dependent backgrounds. String theory can be used reliably to calculate scattering processes involving gravity only for low energies, $E$, in particular $E \ll 1 / g_{s}$, where $g_{s}$ is the string coupling. If $E \ll 1 / l_{s}$ as well, where $l_{s}$ is the string length scale, string theory is validated by reproducing the known results from General Relativity (GR). When one studies problems which are unresolved in GR, such as the propagation through space-like singularities, one would actually wish that the string theory analysis will deviate, in a subtle but significant manner, from that of GR. In this way string theory will fulfil its duty and resolve outstanding problems in GR while adhering to the correspondence principle.

Is such a behavior possible at all? This actually is the case for the propagation of strings in the presence of some time-like singularities. Perturbative and non-perturbative effects do modify in a subtle way the behavior obtained in a GR framework. A story in the Talmud helps exemplify the manner in which extended objects modify point particle problems. During a seminar the following question came up: Assume one finds a pigeon somewhere, to whom does it belong? The rule is, finders-keepers, as long as the pigeon

\footnotetext{
${ }^{1}$ Based on talks given by Amit Giveon and Eliezer Rabinovici at the 35'th International Symposium Ahrenshoop on "The Theory of Elementary Particles," at the Akademie Berlin-Schmöckwitz, 26-30 August 2002; to appear in the proceedings.

$\S$ E-mail : giveon@vms.huji.ac.il ${ }^{1}$, eliezer@vms.huji.ac.il ${ }^{2}$, asever@phys.huji.ac.il ${ }^{3}$
} 
is found further away than some determined distance from the entrance to an owned pigeon-hole. This seems rather well defined for point pigeons. A student raised his hand and asked: "what if the pigeon exhibits its extended object nature? that is what if one of its legs is nearer than the prescribed distance to the pigeon-hole but the other is further away?" The student was actually ejected from the Yeshiva for asking this question.

In this talk we give a short review on some of the recent work on strings propagating in time-dependent backgrounds. The talk has the following structure. It starts by reviewing various problems rising in a GR study of compact cosmology. This follows by suggesting a stringy point of view on several of these problems. Exact stringy time-dependent backgrounds are discussed. Special attention is given to the algebraic, geometrical and dynamical aspects of coset constructions. The results obtained allow a new point of view on the problems and are reviewed. The difficulties encountered and some of the open problems are discussed. These include a study of singularities; some of the studies in these proceedings are new (presented in section 7 ).

\section{Some Questions regarding Cosmologies in General Relativity}

We start by discussing some of the generic problems encountered by studying a universe, compact in space, using GR methods.

- Constructing Time-Dependent Solutions:

It is not immediate to construct time-dependent solutions of the equations of GR that contain space-like singularities. We will describe how such solutions can be generated. We will mainly focus on backgrounds which are in addition exact string backgrounds. These will be coset models and orbifolds.

- Observables:

One needs to describe what are the appropriate observables to be measured in the system. Moreover the universe could even be so small as to disallow the installation of a classical measuring device necessary for a quantum measurement.

- Cauchy data/Singularities:

In GR one studies the evolution of the system as a function of Cauchy data. In the presence of singularities it may be unreliable to impose the data and/or the boundary conditions at the singularity itself. One would rather impose boundary conditions in regions of space in which the couplings and curvature are small. In such regions the semi-classical picture may be a useful guide. Where are such regions? Given appropriate boundary conditions, it is instructive to follow the scattering of a probe from the space-like singularity and to calculate the amount reflected and transmitted. One would like to know if the question has a precise meaning. Perhaps all the information can be encoded in the region up to the singularity. For black holes there are claims that it is enough to consider the space-time up to the horizon of the black hole [1].

- Entropy [2]:

In the absence of a global time-like Killing vector it is not at all clear how to define states in GR and how to count them. In de-Sitter space, arguments have been presented that the total "number of states" is actually finite [3]. This is done by 
analogy to the classical entropy in a black hole. Is there a stringy microscopic estimate of that entropy?

- (In)Stability of the Cosmology $[4,5]$ :

In GR it is known that there are circumstances under which the presence of a speck of dust can totally disrupt the global geometry. Does string theory have anything to add to this?

\section{$3 \quad$ Stringy Attempts to Address the Questions}

- Constructing Time-Dependent Solutions:

Time-dependent backgrounds containing space-like singularities have been constructed and studied using several methods.

1. Exact String Solutions [6, 7, 8, 9, 10, 11, 12, 13, 14, 15, 16]:

Exact conformal field theories which contain space-like singularities have been constructed. The advantage of such constructions is that they are exact as far as $\alpha^{\prime}$ corrections are concerned. This allows for example the exact calculation of vertex operators using algebraic methods without the need to resort to a classical geometrical picture. This was done for a class of coset models $G / H \times M$ where $M$ is an attendant manifold to a time-dependent four dimensional cosmology. This was also done for a class of orbifolds of Minkowski space. The results are mainly for the tree approximation as far as string perturbation theory is concerned. We review in some detail the results of such constructions.

2. S-Branes:

These are proposed solutions which are supposed to be in some sense Dirichlet branes with a fixed coordinate in the time direction. In a manner the idea that such objects may indeed be exact solutions are inspired by the presence of the Euclidean instantons. For a review and references see J. Walcker's talk.

3. Holography Inspired Relations:

A non-perturbative description of the properties of strings in time-dependent backgrounds is desirable. Such a description was made possible in those systems in which the property of Holography was essentially shown to exist. If one assumes that topology is valid also in some time-dependent background settings, one may wish to claim that this holographic description is obtained by simply making the world-volume of the holographic dual theory be the appropriate boundary time-dependent background. The non-perturbative description of the string theory being essentially a boundary field theory on an appropriate timedependent background. The analysis of this field theory is yet to be done. Aspects of holography and its applications are discussed in V. Balasubramanian's talk, (see references there).

4. Solutions Obtained by Double Wick Rotating GR Solutions which Contain TimeLike Singularities [17]:

These are solutions to Einstein's equations but are not exact string backgrounds. They are useful to study phenomena such as particle creation which occurs in time-dependent backgrounds as well as to capture important features of the stability of the system. 
5. RG Flow Induced by Unstable Configurations [18]:

Tachyonic decay in the open string sector of D-branes and in some closed string settings can also be formulated as a time-dependent background. It has been suggested that this has implications in realistic cosmologies. It is a useful setup for studying time independent backgrounds. These important studies are not reviewd here.

- Observables:

One needs to describe what are the appropriate observables to be measured in the system. In the string theory these may be the BRST-invariant operators. Moreover in string theory the S matrix is the observable. In a compact universe it is not clear where to place the asymptotic scattering states. This is resolved in a class of models in a rather surprising manner. Some exact string backgrounds turn out to contain in addition to compact time-dependent cosmologies also static regions which extend all the way to spacial infinity and are called "whiskers" (see figure 1). ${ }^{2}$

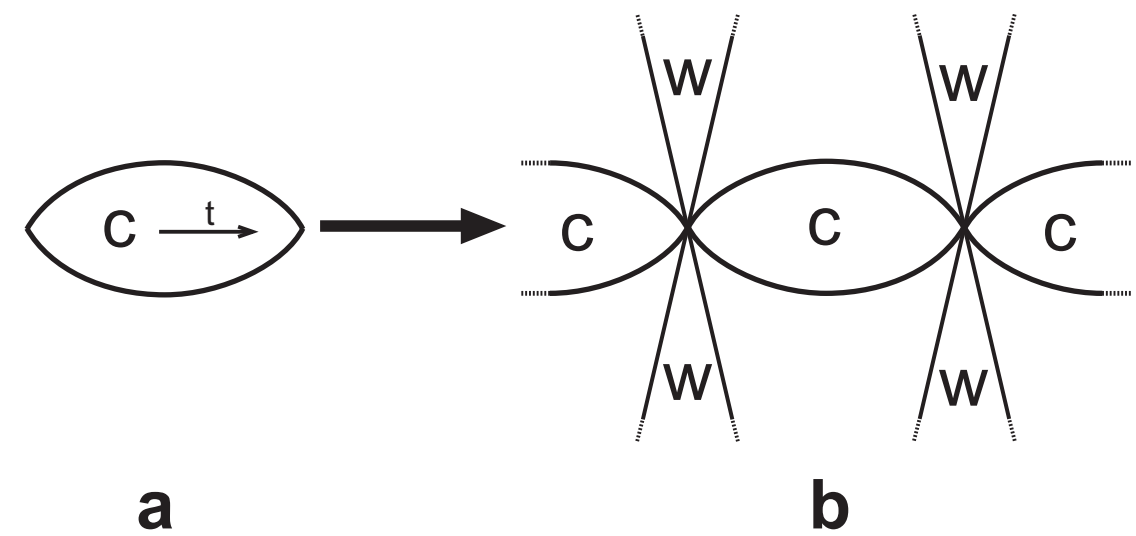

Figure 1: a: A compact cosmology (C) in GR. b: The same compact cosmology repeats itself in string theory and comes with additional regions - whiskers $(\mathrm{W})$.

Moreover, the system is weakly coupled at the boundary of these regions. The accelerators and detectors can thus be placed there to produce and detect scattering states. There is a possible fly in the ointment; these regions contain closed time-like curves and may contain in some cases time-like singularities as well.

- Cauchy data/Singularities:

Once a time-independent weakly coupled region exists it is natural to use it to enforce boundary conditions and to give the Cauchy data. The behavior of the system at the singularity is determined by the behavior at these better understood regions. In fact, the reflection coefficient of a given partial wave vertex operator can be calculated and is found to be smaller than one [6]. The scattering can be shown to be unitary. For the orbifold backgrounds also the scattering of two vertex operators has been calculated $[14,15]$. The results indicated problems; these are discussed later.

- Entropy:

\footnotetext{
${ }^{2}$ In section 7 we shall discuss instead examples where the static regions are compact and singular.
} 
In string theory the entropy can be estimated by counting the BRST-invariant states. In a class of models the number is significantly depleted relative to other non-cosmological systems [6].

- (In)Stability of the Cosmology [19]:

There are at least two types of stability problems in such systems: short and long time scale problems. Consider first the short time scale problem. In the presence of a space-like singularity, classically the energy density increases without bounds as the projectile approaches the singularity which is concentrated at zero volume; this would be suggested even if the energy is conserved. If it is blue shifted as well as one approaches the singularities the situation would only be worsened. In the orbifold models this was pointed out from several points of view which had the common feature of closed time-like curves and space-like singularities. A prototype of such a universe is the Misner Universe. Its metric is given by

$$
d s^{2}=-d t^{2}+t^{2} d x_{1}^{2}+d x_{2}^{2}+d x_{3}^{2}
$$

with the identification:

$$
\left(t, x_{1}, x_{2}, x_{3}\right)=\left(t, x_{1}+n a, x_{2}, x_{3}\right)
$$

After a change of coordinates:

$$
\begin{aligned}
& y_{0} \equiv t \sinh \left(x_{1}\right) \quad y_{2} \equiv x_{2} \\
& y_{1} \equiv t \cosh \left(x_{1}\right) \quad y_{3} \equiv x_{3}
\end{aligned}
$$

the metric becomes:

$$
d s^{2}=-d y_{0}^{2}+d y_{1}^{2}+d y_{2}^{2}+d y_{3}^{2}
$$

with the identification (see figure 2):

$$
\left(y_{0}, y_{1}, y_{2}, y_{3}\right)=\left(y_{0} \cosh (n a)+y_{1} \sinh (n a), y_{0} \sinh (n a)+y_{1} \cosh (n a), y_{2}, y_{3}\right)
$$

The identification has a fixed submanifold given by:

$$
\left(y_{0}, y_{1}, y_{2}, y_{3}\right)=\left(0,0, y_{2}, y_{3}\right)
$$

The resulting orbifold contains close time-like curves. A free field type calculation of the expectation value of the energy-momentum tensor as a function of the distance to the above singularity, $t_{s}$, is singular and given by [5]:

$$
\left\langle T_{\mu \nu}\right\rangle=-i \lim _{y_{1} \rightarrow y_{0}}\left(\frac{2}{3} \nabla_{\mu} \nabla_{\nu} \ldots\right) G^{\mu \nu}\left(y_{0}, y_{1}\right)=\frac{a}{t_{s}^{4}} \operatorname{diag}(1,-3,1,1)
$$

where $a$ is a system dependent constant. We will discuss more aspects of this singularity. As mentioned, the system is also threatened by a long time scale instability. This may occur for systems with a discrete spectrum and a finite number of "states" or at least a finite thermal entropy. Compact cosmologies may be systems of this nature. For the AdS case the instability starts when the contribution of the eternal black hole masterfield, embedded in AdS, is superceded by that of non-dominant thermal AdS masterfield [20]. Recall that these two masterfields have different topologies [21]. These instabilities were indicated from GR arguments. On the other hand, in time-independent string backgrounds we are familiar with two types of instabilities: perturbative ones - tachyons - which face no barrier in their decay, and non-perturbative instabilities (such as the formation of small black holes from flat Minkowski space at finite temperature) which do need to overcome a barrier. 


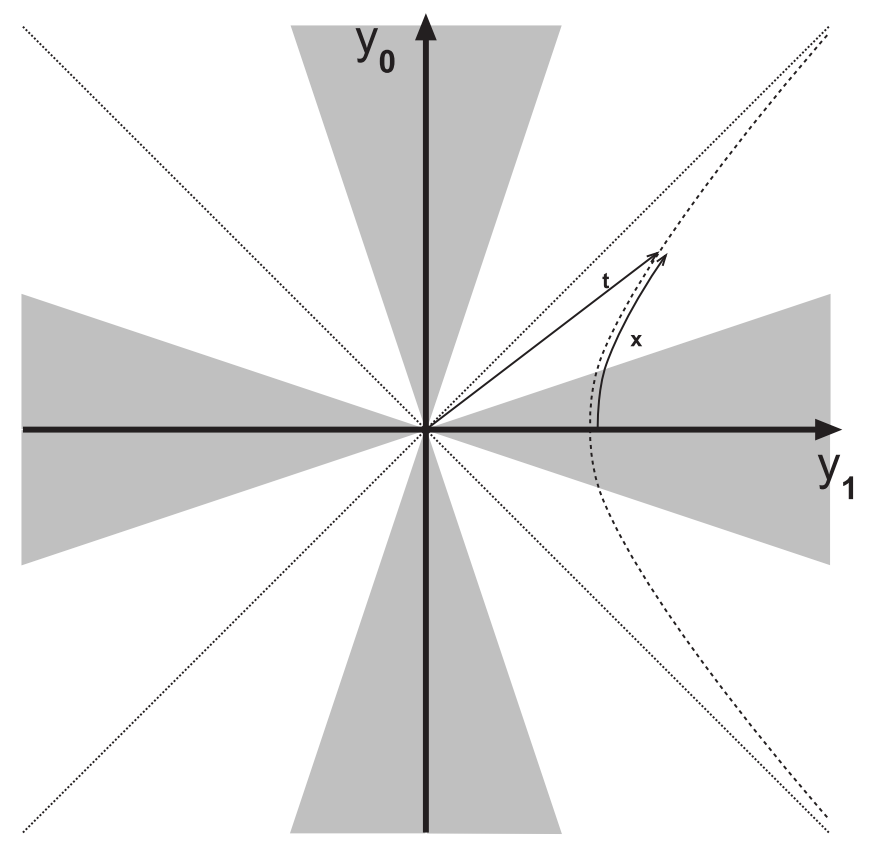

Figure 2: The extended Misner universe; a fundamental domain is marked.

\section{Exact Time-Dependent Backgrounds}

Two types of exact time-dependent backgrounds containing space-like singularities have been considered. Both have bosonic and fermionic versions with the respective central charge $c=26$ and $c=15$. The first type are $G / H$ cosets. In particular the coset $[6,13]$ :

$$
\frac{S L(2, \mathbb{R})_{k} \times S U(2)_{k}}{U(1) \times U(1)} \times M
$$

In this case an identification is done on the group elements of $G$ by an operation with group elements of $H$ in the following manner:

$$
G / H: \quad\left(g, g^{\prime}\right) \rightarrow\left(e^{\rho \sigma_{3}} g e^{\tau \sigma_{3}}, e^{i \tau \sigma_{3}} g^{\prime} e^{i \rho \sigma_{3}}\right), \quad\left(g, g^{\prime}\right) \in S L(2) \times S U(2)
$$

The second type are orbifolds. A particular identification is given by [14]:

$$
G / \Gamma: \quad\left(x^{+}, x, x^{-}\right) \rightarrow\left(x^{+}, x+2 \pi x^{+}, x^{-}+2 \pi x+\frac{1}{2}(2 \pi)^{2} x^{+}\right)
$$

$G$ is actually $1+2$ Minkowski space in this case. Both identifications have fixed points but they are of a different nature. The form of the manifold (10) is $\frac{R^{1,2}}{\Gamma} \times M$, where $\Gamma$ is a finite parabolic subgroup of the Poincaré symmetries of Minkowski space. The metric of the $\frac{R^{1,2}}{\Gamma}$ factor is:

$$
d s^{2}=-2 d x^{+} d x^{-}+d x^{2}
$$

with the identification (10). The fixed plane is $\left(0,0, x^{-}\right)$; it is the origin of the light-like orbifold singularity. The surface $x^{+}=0$ has closed null-like curves.

The metric of (11), (10) can be written as:

$$
d s^{2}=-2 d y^{+} d y^{-}+\left(y^{+}\right)^{2} d y^{2}, \quad y \sim y+2 \pi n
$$


where

$$
y^{+}=x^{+}, \quad y=\frac{x}{x^{+}}, \quad y^{-}=x^{-}-\frac{1}{2} \frac{x^{2}}{x^{+}}
$$

This is similar to the Misner space (1), (2). At $y^{+}=0$ there is a light-like big bang/crunch singularity, and near $y^{+}=0$ the space is non-Hausdorff.

Different orbifolds discussed in the literature $[10,9,14,19,22,23]$ are, for instance: $t \rightarrow-t, t \rightarrow t+2 \pi$, and other parabolic and hyperbolic orbifolds. In the above cases $G$ was taken to be the Poincaré symmetry group of the $d+1$ Minkowski space, and thus is a known CFT. Different types of orbifolds are the BTZ black holes in which case $G$ is the group manifold $S L(2, \mathbb{R})$. The orbifolds $G / \Gamma$ have physical twisted sectors in most cases.

Some of the systems are supersymmetric. Supersymmetry prevents among other things particle production, removes tadpoles, tachyons and makes the one loop "cosmological constant" vanish. At this stage it is not clear if supersymmetry really plays an important role in allowing a string perturbative analysis of the theory. The main positive result was that for a class of these supersymmetric backgrounds the two and three point functions were calculated and were found to be well behaved despite of the semiclassical space-like geometric singularity. The worse is yet to come: divergences appear in higher point, string tree-level correlators, in some models [14].

We now turn to discuss some general aspects of coset CFTs, and coset models leading to time-dependent backgrounds in particular.

\section{Algebraic, Dynamic and Geometrical Aspects of Coset back- grounds}

1. Algebraic Aspects of Coset Models [24]:

The special feature of the algebraic treatment of coset CFTs is that it allows one to obtain the exact vertex operators and in principle any $n$-point function without the need to worry about the semiclassical geometrical description of the system. Of course, singularities in the geometrical description will either be cured or come back to haunt us in the algebraic description. In order to appreciate the string backgrounds constructed out of cosets consider first the WZW models. From an algebraic point of view one is given a group $G$ from which one can construct first an affine Lie algebra and then representations of the Virasoro algebra. Algebraically it is simpler to consider worldsheet chiral algebras. In particular the operator product expansion (OPE) defining the affine Lie algebra of $G$ is:

$$
J^{a}\left(z_{1}\right) J^{b}\left(z_{2}\right)=\frac{k \delta^{a b}}{z_{12}^{2}}+\frac{i f_{c}^{a b} J^{c}\left(z_{2}\right)}{z_{12}}
$$

where $J$ are chiral worldsheet currents and $k$ is the level of the affine algebra. A similar OPE holds for the anti-chiral currents, $\bar{J}^{a}$. The chiral component of the energy-momentum tensor is constructed out of the currents $J$ by:

$$
T \equiv \frac{: J^{a} J_{a}:}{k+h}
$$

where $h$ is the dual coxeter number of the Lie group. For example, $h=k+2$ for $S U(2)$ and $h=k-2$ for $S L(2, \mathbb{R})$. The OPE of two T's is:

$$
T\left(z_{1}\right) T\left(z_{2}\right) \sim \frac{c}{z_{12}^{4}}+\frac{2 T\left(z_{2}\right)}{z_{12}^{2}}+\frac{\partial_{2} T}{z_{12}}
$$


where $c=\frac{k \cdot \operatorname{dim} G}{k+h}$ is the Virasoro central charge. For $S U(2): c=\frac{3 k}{k+2}$, and for $S L(2, \mathbb{R}): c=\frac{3 k}{k-2}$. The algebraic coset $G / H$ also induces a representation of the Virasoro algebra. Given a group $G$ with a subgroup $H$, one constructs the following energy-momentum tensor:

$$
T_{G / H} \equiv T_{G}-T_{H}=\frac{: j j:_{G}}{k_{G}+h_{G}}-\frac{: j j:_{H}}{k_{H}+h_{H}}
$$

where $c(G / H)=c(G)-c(H)$. For example, $c(S U(2) / U(1))=\frac{3 k}{k+2}-1, c(S L(2) / U(1))=$ $\frac{3 k}{k-2}-1$ (when the $U(1)$ is null replace $-1 \rightarrow-2$ ). Can a physical meaning be attributed to the minus sign responsible for the decrease in the central charge? The answer is yes and we will now turn to discuss it.

2. The Physics of coset models:

We will describe the coset models as an infra-red limit of two dimensional confining gauge theories. To obtain that consider first a conformal field theory: $L=L_{C F T}$ with some Virasoro central charge $c_{U V}$. Next add to the theory a relevant operator, or add an operator which is classically marginal but not truly marginal. This operator should have an asymptotically free flow. The resulting Lagrangian is: $L=L_{C F T}+$ $\sum_{i} g_{i} O_{i}$ ("relevant"). The ultra-violet (UV) limit of the theory is described by the Lagrangian $L_{C F T}$. The infra-red (IR) limit of any field theory is a conformal theory. Generically it has a vanishing central charge, $c_{I R}=0$. In a unitary theory: $c_{I R}<c_{U V}$ [25]. As a first example consider as the UV theory a free Dirac fermion and modify it by adding the relevant mass operator. The UV Lagrangian is:

$$
L_{C F T}=\bar{\psi} \not \partial \psi
$$

This system has a $c=1$ Virasoro central charge. The full Lagrangian is not conformal and given by:

$$
L=\bar{\psi} \not \partial \psi+m \bar{\psi} \psi
$$

This is an exactly solvable system. It has a mass gap which becomes infinite in the extreme IR limit. Only the ground state(s) survive in this limit. The topology of the worldsheet may allow several vacua which may constitute a topological field theory. In the absence of non-trivial topology there is a single vacuum state. Be that as it may, the IR theory has $c=0$ which is smaller than $c=1$. In the second example one gauges the full global $U(1)$ that the free Dirac Fermion Lagrangian posses. This is described by:

$$
L=\bar{\psi} \not \partial \psi+J_{\mu} A^{\mu}+\frac{1}{g^{2}} F_{\mu \nu}^{2}
$$

where $A^{\mu}$ is the vector potential. The coupling is dimensionfull, with dimensions of a mass, thus the gauging results in adding a relevant perturbation to the free Dirac Fermion. This is the massless Schwinger model. The potential between two heavy charged sources is confining, $V(r) \sim r$. In fact, with massless Fermions the theory (super) confines. Only one bound state is formed and it is a local object having for example no dipole moment (in the case of massive Fermions they do have a dipole moment and several bound states may form). In the extreme IR the gauge coupling becomes infinite and only the ground state remains (up to perhaps topological issues mentioned before). To see this explicitly recall that the bosonized version of the Lagrangian is:

$$
L=\frac{1}{2}\left(\partial_{\mu} \sigma\right)^{2}+\frac{m^{2}}{2} \sigma^{2}
$$


where $\sigma$ is a superlocal bound state - a free massive scalar with $m \sim g$. In the IR $g, m \rightarrow \infty$ hence all excitations decouple. It leaves behind only the vacuum state(s), a $c=0$ IR theory. As in the massive Dirac Fermion case $c_{U V}=1 \rightarrow c_{I R}=0$. Moreover, in the IR $g \rightarrow \infty$, thus in the IR the term $\frac{F_{\mu \nu}^{2}}{g^{2}}$ disappears from the Lagrangian (20), leaving just the terms linear in the gauge potential. The potential is reduced to a Lagrange multiplier and this is the actual Lagrangian representation of the coset model:

$$
L_{I R}=L_{C F T}+J_{\mu} A^{\mu}
$$

The third example is the Lagrangian representation of a special class of coset models. For simplicity we consider here the group $G$ to be $S O(N)$. In that case the $W Z W$ model can be expressed as a free Fermion system. Its Lagrangian is:

$$
L_{C F T}=L_{\text {Free-Fermions }}(G)
$$

Consider gauging the full $S O(N)$ group. This gives:

$$
L=L_{F F}+J_{\mu}(G) A^{\mu}(G)
$$

This is the Lagrangian of the coset $G / G$ which has a vanishing Virasoro central charge $c=0$. All the particles obtain a mass as confined bound states which decouple in the extreme IR. The last example is the general coset [26]. It is obtained by gauging a subgroup $H$ of $G$ :

$$
L=L(G)+J_{\mu}(H) A^{\mu}(H)
$$

This is a schematic description of WZW system of the group $G$ gauged by a subgroup $H$. The exact form appears below. The resulting central charge decreases as: $c(G / H)=c(G)-c(H)$. The decrease in central charge is traced to the partial confinement, that of $H$ singlets.

3. Geometrical Aspects of Coset Models:

The geometrical treatment starts, as the algebraic one did, by first considering the affine Lie symmetry. One writes down a Lagrangian which has a conformal as well as affine symmetry. That Largangian leads to a Hamiltonian which once diagonalized has eigenfunctions which form representations of both the conformal group and the affine Lie algebra. One is familiar in string theory with a geometrical description of the target space in which the string propagates by a non-linear $\sigma$ model (NLSM). Given the group $G$ one may consider writing down the appropriate NLSM by parameterizing the group member $g \in G$ as $g=e^{i x^{\alpha} T_{\alpha}}$ and forming the NLSM $L=G_{\alpha \beta} \partial x^{\alpha} \bar{\partial} x^{\beta}$ where $G_{\alpha \beta}$ is the appropriate metric on the group manifold. This however is usually not a CFT. In fact, the beta-function of the couplings $G_{\alpha \beta}(x)$ obey (to leading order in $\alpha^{\prime}$ ):

$$
\begin{aligned}
>0 & \text { For a non - compact group the theory is IR free } \\
R_{\mu \nu} \sim \beta_{G_{\mu \nu}}<0 & \text { For a compact non - abelian group the theory is UV free } \\
& =0
\end{aligned}
$$

To get a CFT for any group $G$ the Lagrangian, $L$, needs to be modified. An antisymmetric field $B_{\alpha \beta}$ is added to the geometric target space description to make the theory conformal (this operation is sometimes called to parallize the manifold). The new Lagrangian is:

$$
L=g^{i j} G_{\alpha \beta} \partial_{i} x^{\alpha} \partial_{j} x^{\beta}+\epsilon^{i j} B_{\alpha \beta} \partial_{i} x^{\alpha} \partial_{j} x^{\beta}
$$


The geometrical description of the coset is given by gauging the subgroup $H$ of $G$. The $H$ locally gauge invarinat Lagrangian is given by:

$$
\begin{aligned}
S[g] & =\frac{k}{4 \pi} \int_{\Sigma} d z^{2}\left[\operatorname{Tr}\left(g^{-1} \partial g g^{-1} \bar{\partial} g\right)+A_{z}^{a} A_{\bar{z}}^{a}+\left(A_{\bar{z}}^{a} \operatorname{Tr}\left(g^{-1} \partial g T^{a}\right)+c . c\right)\right] \\
& +\frac{i k}{12 \pi} \int_{B} \operatorname{Tr}\left(g^{-1} d g\right)^{3}
\end{aligned}
$$

This is the full form of the gauged Lagrangian. The NLSM describing the coset can be obtained by integrating out the gauge fields, $A_{\mu}^{a}$. The integral is a guassian one. Actually one needs to take note of the measure as well. This results in a modified NLSM, the metric and the antisymmetrical tensor change and a new background field - the dilaton $\Phi$ - emerges: $(G, B)_{W Z W} \rightarrow\left(G^{\prime}, B^{\prime}, \Phi\right)_{\text {coset }}[27]$. This geometric description is exact only for large $k$. Suggestions for obtaining "exact" geometrical backgrounds have been put forward. In any case the geometric data provides a semiclassical picture. The algebraic treatment allows precise calculations. In abelian quotients the two and three point functions are identical to their corresponding ones in $G$. Those functions are smooth on the group and therefore also on the coset even if the coset has singularities. This ends the general discussion we now turn to the background at hand.

\section{Cosmology and Whiskers [6, 13]}

From the algebraic point of view the system is the coset

$$
\frac{S L(2, \mathbb{R})_{k} \times S U(2)_{k}}{U(1) \times U(1)} \times M
$$

As the original motivation is to construct a compact manifold with space-like singularities one is led to examine the semi-classical geometry induced by the algebraic coset. This geometry includes time-dependent cosmological regions and whiskers, as mentioned. In the cosmological regions the metric, dilaton and B field are [6]:

$$
\begin{gathered}
\frac{1}{k} d s^{2}=-d \theta_{1}^{2}+d \theta^{\prime 2}+\frac{\cot ^{2} \theta^{\prime}}{1+\tan ^{2} \theta_{1} \cot ^{2} \theta^{\prime}} d \lambda_{-}^{2}+\frac{\tan ^{2} \theta_{1}}{1+\tan ^{2} \theta_{1} \cot ^{2} \theta^{\prime}} d \lambda_{+}^{2} \\
B_{\lambda_{+}, \lambda_{-}}=\frac{k}{1+\tan ^{2} \theta_{1} \cot ^{2} \theta^{\prime}} \\
\Phi=\Phi_{0}-\frac{1}{2} \log \left(\cos ^{2} \theta_{1} \sin ^{2} \theta^{\prime}+\sin ^{2} \theta_{1} \cos ^{2} \theta^{\prime}\right)
\end{gathered}
$$

where $\lambda_{ \pm} \in[0,2 \pi)$, and $\theta_{1}$ and $\theta^{\prime}$ vary in the interval $\left[0, \frac{\pi}{2}\right]$. In the whiskers: ${ }^{3}$

$$
\begin{gathered}
\frac{1}{k} d s^{2}=d \theta_{2}^{2}+d \theta^{\prime 2}+\frac{\cot ^{2} \theta^{\prime}}{1-\tanh ^{2} \theta_{2} \cot ^{2} \theta^{\prime}} d \lambda_{+}^{2}-\frac{\tanh ^{2} \theta_{2}}{1-\tanh ^{2} \theta_{2} \cot ^{2} \theta^{\prime}} d \lambda_{-}^{2} \\
B_{\lambda_{+}, \lambda_{-}}=\frac{k}{1-\tanh ^{2} \theta_{2} \cot ^{2} \theta^{\prime}}
\end{gathered}
$$

\footnotetext{
${ }^{3}$ The geometric data obtained is valid in the large $k$ limit. For the bosonic string there are known $1 / k$ corrections [12, 28]. The exact background sometimes has a different singularity structure. For fermionic strings, the semiclassical background is expected to be a solution to all orders in $1 / k$. The dilaton $\Phi$ is normalized such that the string coupling is $g_{s}=e^{\Phi}$.
} 


$$
\Phi=\Phi_{0}-\frac{1}{4} \log \left(\cosh ^{2} \theta_{2} \sin ^{2} \theta^{\prime}-\sinh ^{2} \theta_{2} \cos ^{2} \theta^{\prime}\right)^{2}
$$

where here $\theta_{2} \in[0, \infty), \theta^{\prime} \in\left[0, \frac{\pi}{2}\right], \lambda_{ \pm} \in[0,2 \pi)$.

The geomerty is somewhat complex, and follows a list of features which play a role in addressing the questions posed in the beginning of the talk:

- Time-Dependent Comological Regions:

The geometry (30) indeed describes a compact cosmological region, it is denoted by $\mathrm{C}$ in figure 1. In fact, the exact string background contains an infinite number of such C regions, (for a partial list of earlier works see [29]). Each C region can be viewed as starting from a mild big bang in which only one of its spacial coordinate directions degenerates, and ends with a mild big crunch where, again, the spacial volume vanishes along a different direction. The manifold thus is not isotropic. The maximal spacial volume of the cosmological region increases with the algebraic level, $k$, of the affine Lie algebra. The source of the infinite sequence of bangs and crunches is that in order to avoid having ab-initio closed time-like curves (CTC) in the manifold, one considers the universal cover of $S L(2, \mathbb{R})$ and not just the group itself. One may pose the set of questions referring to these compact regions. The surprise is the appearance of additional regions: the whisjers, denoted by $\mathrm{W}$ in figure 1. These regions extend all the way to different spacial infinities. Their emergence sheds a new light on the set of questions.

- The Whiskers Regions and the S Matrix Problem [6]:

The $\mathrm{W}$ regions have infinite extent and moreover their metric is time-independent. String theory has thus provided a place to build accelerators so one can measure the $\mathrm{S}$ matrix. The common wisdom claims one should be able to do it in string theory and indeed one can. The whiskers also contain time-like submanifolds which are singular and CTCs. Their significance needs to be studied.

- Observables and Entropy [6]:

The BRST exact operators are natural candidates to be the observables. Their number could be defined as the entropy of the system. The analysis shows that the number of states is infinitely depleted relative to the number of states in the $A d S_{3}$ case. In the latter case the ten-dimensional string theory can be represented by a two-dimensional dual conformal field theory. The case at hand contains even fewer states [6]. Examples of observables that were calculated are the vertex operators in the theory. One of them, $\mathrm{U}$, corresponding to a $\delta$ function normalizable operator, is given by $[6,30]$ :

$$
V=U D f
$$

where $D$ is the $S U(2)$ part of the vertex, $f$ is the internal $M$ part, and $U$ is given by:

$$
U\left(m, m^{\prime} ; j, \epsilon ; g\right)=K_{++}-\frac{\sin \left(\pi\left(j+i m^{\prime}\right)\right)}{\sin (\pi(j+i m))} K_{--}
$$

where $m, m^{\prime}, j$ are the usual $S L(2)$ numbers related to the energy and momenta in target space (see below), and $K_{++}, K_{--}$are defined in [31]. What is important here is that $U$ has the following asymptotic form on the boundary of the whiskers:

$$
U\left(E_{ \pm} ; j ; \theta_{2} \rightarrow \infty\right) \sim e^{2 i E_{-} \phi} e^{-\theta_{2}}\left[e^{2 i\left(E_{+} t+p_{s} \theta_{2}\right)}+R\left(j ; m, m^{\prime}\right) e^{2 i\left(E_{+} t-p_{s} \theta_{2}\right)}\right]
$$


where

$$
E_{ \pm}=\frac{1}{2}\left(m \pm m^{\prime}\right), \quad j=-\frac{1}{2}+i p_{s}
$$

From this form one can extract the reflection coefficient of each partial wave. Its square is given by:

$$
1 \geq|R|^{2}=\frac{\cosh \left(2 \pi E_{+}\right)+\cosh \left(2 \pi\left(p_{s}-E_{-}\right)\right)}{\cosh \left(2 \pi E_{+}\right)+\cosh \left(2 \pi\left(p_{s}+E_{-}\right)\right)}
$$

The theory is unitary. This results from the fact that string theory on $S L(2, \mathbb{R})$ is unitary and has similar lower point functions. The interpretation of that part of the vertex operator as a reflection coefficient was possible after choosing the appropriate boundary conditions. The system is defined far away from the singularities in a weak coupling regime as one would indeed prefer.

- Bounday Conditions and Possible Crossing of the Singularities:

Once the boundary conditions are set in a weakly coupled region, the vertex operator is fixed everywhere. This includes the regions following the particular big bang and the following big crunches and big bangs. A projectile starting in a definite partial wave in one of the whiskers ends up also in other whiskers. Again, unitarity of string theory on $S L(2, \mathbb{R})$ is responsible for the unitarity when summed over all the boundaries of the whiskers. One resolution of the problem of crossing the singularity would be that the singularity was there only semi-classically. Had the singularities had no algebraic origin that would be likely. However, one can associate some of the singularities which are manifest in the geometrical formulation with fixed points of the algebraic identifications implied in constructing the coset. Other are not and can be removed as will be discussed later.

- Holographic Aspects:

At this stage one would still require that holography be derived for each case and not be added to the axioms of a theory of gravity. There is thus no guarantee that this background has a holographic description, nevertheless one can observe several primodial seeds of this feature. The universe seems to be divided in cosmologies and whiskers. Holographic screens, if they have a meaning, are likely to be set at the asymptotic boundaries of the whiskers. "We" on the other hand are presumably situated in the cosmological parts. From the point of view of the bulk theory there are several types of representations of $S L(2, \mathbb{R})$ which play a role in the mapping between the boundary and the bulk theories. The discrete representations of $S L(2, \mathbb{R})$ reflect normalizable states which live near the cosmological region, the information about them may be encoded in the non-normalizable operators from that series which extend to the boundary. The principal continuous representations give more global information about the physics in the bulk and are related on the boundary to the $\delta$ function normalizable operators. The system seems to have an infinite number of screens. Thus one may compose the dual theory out of an infinite entangled Hilbert spaces, the wave function being a product of an infinite number of correlated states (a general claim on this issue was made in [20]). Recently, in the BTZ black hole case a reduction in the number of effective boundaries was indicated [32]. This could be however related to special scattering properties of the black hole. As mentioned strings propagating on $A d S_{3}$ times an attendant manifold do have duals [33]. One may expect that the same is true after gauging and appropriate compensation of the 
central charge. Such a non-perturbative definition could be of value, and studies of the simpler BTZ black hole case were done. To summarize the positive results, the study has allowed the explicit calculation of some interesting properties of strings in the presence of cosmology and has offered some surprising ways to resolve long standing problems in GR. There are however also many open issues.

- Back Reaction and Other Open Issues:

As described earlier the cosmological background is exactly of such a complicated nature that it could be destabilized by a speck of dust. The worry that string perturbation theory would invalidate the supposedly exact calculation at tree level arises because the various exact solutions have CTC and less generic closed nulllike curves, in addition to the space-like singularities. We have reviewed the field theory calculation pointing at a singular energy density at the singularity. The hope was that a string probe smears and thus heals the singularity. The analysis of the lower point functions gives indeed finite answers. This is not the case however for the four point function in one of the orbifold cases; it diverges [14]. The source of that divergence is that the Hamiltonian of the system does not commute with the projection operator of the orbifold construction. As all states must be singlets of that projection, the allowed states can not be eigne-states of energy. There is no way to construct a solely low energy scattering probe. The state needs to contain higher energy components. The question becomes, what is the weight of these high energy components? For the original case it was found that in fact all components had equal weight and thus the average energy of the projectile was infinite, leading to a breakdown of string perturbation theory. In other cases a control parameter was found that allowed to obtain non-singular scattering [7, 15, 23]. For the singularity in the coset the four point function has not yet been calculated exactly. On top of that, non-perturbative effects, such as black hole formation in a region where GR calculations are reliable also threaten the validity of perturbation theory [34]. Preliminary results for the BTZ black hole case did not detect a singularity [32]. The coset case has actually many similarities to the BTZ case [7]. However, these results are not yet conclusive and the issue remains open. The various theories can be studied in a world-volume supersysmmetic version. Some orbifolds can be arranged to be supersymmetric also in target space. As mentioned, the importance of this is not yet clear. Also long strings and various thermodynamical aspects have not yet been studied. There has been some initial work on D-brane probes. The branes probe shorter distances and may offer an additional view on how strings experience the singularities. An uninvited feature emerged both in the compact cosmological backgrounds and several of the orbifold constructions. The systems have regions which contain CTCs. The first negative associations with such curves is that they allow the violation of causality. In the gauged models the origin of the CTCs is the compact $S U(2)$ manifold. Gauge invariance does however select only vertex operators which are single valued. The history they represent repeating itself. In fact the depletion of the number of states has the same origin and is welcome. One may suggest that the two are correlated. The reduction of degrees of freedom going hand in hand with those few states for which causality, as well as other properties, remain intact. In GR CTCs also lead to accumulation of very large energies. In some cases it clearly invalidates perturbation theory taking away the value of supposedly exact solutions. One is thus led to analyze the singularity structure in even more detail. One considers control parameters to study the singularities one of them is 
the radius, $R$, of an extra fifth dimensions (Such extra parameters can be obtained, for instance, by $O(d, d)$ rotations as in $[11,35]$ (for a review, see [36])).

\section{Example: 2-d Cosmology}

In the previous sections we reviewed several examples of exact time-dependent backgrounds in string theory. In particular, four dimensional cosmologies based on Abelian quotients of $S L(2) \times S U(2)$ were studied in detail in [6] and discussed in previous sections. In ref. [7] an Abelian gauge field was turned on by considering a family of Abelian quotients of $S L(2) \times S U(2) \times U(1)$. The presence of the background gauge field changes the structure of the singularities. For instance, a big bang/crunch curvature singularity and the time-like domain wall attached to it can be "pushed" towards the boundary of the whisker by turning on such a gauge field, leaving behind a BTZ-like singularity (for details, see [7]). The fact that such a gauge field background is still described within the context of an exact CFT $-S L(2) \times S U(2) \times U(1) / U(1)^{2}$ - allows to study some properties of the theory, discussed in previous sections, along the lines of [6]. For instance, it was shown that uncharged incoming waves from a whisker can be fully reflected if and only if a big bang/crunch singularity exists, from which it is scattered.

In ref. [16], a two dimensional cosmology based on an Abelian quotient of $S L(2)$ with a BTZ identification was studied in detail along the lines of [6]. In order to understand better the singularity in that space we turn on an Abelian background gauge field by considering instead an Abelian quotient of $S L(2) \times U(1) / Z$. Below we shall mainly consider the geometry of the two dimensional time-dependent backgrounds obtained in this way. The consideration of observables in such geometries can be easily obtained along the lines of $[6,7,16]$.

Explicitly, in this example we construct a family of 3-dimensional time-dependent backgrounds by gauging the WZW model of the 4-dimensional $S L(2, \mathbb{R})_{k<0} \times U(1)$ group manifold by a family of non-compact time-like $U(1)$ subgroups. Then by taking a small constant radius of the $U(1)$ part we obtain a two dimensional cosmology via the KaluzaKlein (KK) mechanism. All two dimensional spaces have: compact static regions, which admit CTC after the BTZ identification, non-compact time-dependent regions that are flat in the asymptotic infinite past or future, a non-trivial dilaton and a background KK gauge field. All regions are generically separated by horizons, which turn into orbifold singularities after the $Z$ identification. This family of backgrounds admits two kinds of singularities: one is generated by fixed points of the gauge group (and lie in the compact region), and the other by fixed points of an orbifold identification (and lie between regions). These backgrounds can also be obtained by $O(1,2) \subset O(2,2)$ rotations along the lines of [37] (for a review, see [36]). In that family, a specific two dimensional space, with a vanishing gauge field, was studied in [16]. In this section we shall concentrate on the geometry of those two dimensional cosmologies with a background gauge field.

Let $(g, x) \in S L(2) \times U(1)$ be a point on the product group manifold where $x \sim x+2 \pi r$ and let $-\kappa \equiv k<0$ be the level of $S L(2, \mathbb{R})$. The $U(1)$ gauge group acts as

$$
\left(g, x_{L}, x_{R}\right) \rightarrow\left(e^{\rho \sigma_{3} / \sqrt{\kappa}} g e^{\tau \sigma_{3} / \sqrt{\kappa}}, x_{L}+\rho^{\prime}, x_{R}+\tau^{\prime}\right) .
$$

Since we gauge only $U(1)$ out of the two right-handed $U(1)$ generators in (41), the two parameters $\left(\tau, \tau^{\prime}\right) \equiv \underline{\tau}$ are not independent but rather are constrained by

$$
\underline{\tau} \equiv \tau \underline{u}
$$


where $\underline{u}$ is some unit real 2 -vector. The left-handed parameters $\left(\rho, \rho^{\prime}\right) \equiv \underline{\rho}$ in $(41)$ depend linearly on the right-handed $\underline{\tau}$ parameters. For an anomaly free gauging this dependence has to take the form

$$
\underline{\rho}=R \underline{\tau},
$$

where the matrix $R$ is an $O(1,1 ; \mathbb{R})$ matrix ${ }^{4}$

$$
R=\left(\begin{array}{cc}
\cosh (\alpha) & \sinh (\alpha) \\
\sinh (\alpha) & \cosh (\alpha)
\end{array}\right)
$$

The gauged action, as in [7], is then defined by

$$
S=S\left[e^{\hat{\rho} \sigma_{3} / \sqrt{\kappa}} g e^{\hat{\tau} \sigma_{3} / \sqrt{\kappa}}\right]+S^{\prime}\left[x+\hat{\rho}^{\prime}+\hat{\tau}^{\prime}\right]-\frac{1}{2 \pi} \int d^{2} z(\partial \underline{\hat{\rho}}-R \partial \hat{\hat{\tau}})^{T}(\bar{\partial} \underline{\hat{\rho}}-R \bar{\partial} \hat{\underline{\tau}}) .
$$

Here, $S[g]$ is the WZW action,

$$
S[g]=-\frac{\kappa}{4 \pi}\left[\int_{\Sigma} \operatorname{Tr}\left(g^{-1} \partial g g^{-1} \bar{\partial} g\right)-\frac{1}{3} \int_{B} \operatorname{Tr}\left(g^{-1} d g\right)^{3}\right],
$$

where $\Sigma$ is the string's worldsheet and $B$ a 3 -submanifold of the group $S L(2)$ bounded by the image of $\Sigma . S^{\prime}[x]$ is

$$
S^{\prime}[x]=\frac{1}{2 \pi} \int_{\Sigma} \partial x \bar{\partial} x
$$

Apart from the constraints (43), $\hat{\rho}$ and $\hat{\underline{\tau}}$ are independent fields. The action (45) is invariant under the gauge transformation (41) for the fields $g$ and $x$ together with the field transformation

$$
\begin{aligned}
& \underline{\hat{\rho}} \rightarrow \underline{\hat{\rho}}-\underline{\rho} \\
& \hat{\underline{\tau}} \rightarrow \underline{\hat{\tau}}-\underline{\tau}
\end{aligned}
$$

provided that the parameters $\rho$ and $\underline{\tau}$ satisfy the relation (43). Using the PolyakovWiegmann identity one sees that the action (45) depends on $\underline{\hat{\rho}}$ and $\underline{\hat{\tau}}$ only through the quantities

$$
\begin{aligned}
& A=\underline{u}^{T} \partial \hat{\hat{\tau}} \\
& \bar{A}=(R \underline{u})^{T} \cdot \bar{\partial} \hat{\rho}
\end{aligned}
$$

The gauged action has then the form

$$
S=S[g]+S^{\prime}[x]+\frac{1}{2 \pi} \int d^{2} z\left[A \overline{\mathbf{J}}^{T} \cdot \underline{u}+\bar{A} \mathbf{J}^{T} \cdot R \underline{u}+2 A \bar{A}^{T}{ }^{T} M \cdot R \underline{u}\right] .
$$

$A$ and $\bar{A}$ are holomorphic and anti-holomorphic gauge fields. $\mathbf{J}^{T}$ and $\overline{\mathbf{J}}^{T}$ are the row vector of currents,

$$
\begin{aligned}
& \mathbf{J}^{T}=\left(\sqrt{\kappa} \operatorname{Tr}\left[\partial g g^{-1} \sigma_{3}\right], 2 \partial x\right) \\
& \overline{\mathbf{J}}^{T}=\left(\sqrt{\kappa} \operatorname{Tr}\left[g^{-1} \bar{\partial} g \sigma_{3}\right], 2 \bar{\partial} x\right)
\end{aligned}
$$

The $2 \times 2$ matrix $M$ in (50) is of the form,

$$
M=\left(\begin{array}{cc}
\frac{1}{2} \operatorname{Tr}\left[g^{-1} \sigma_{3} g \sigma_{3}\right] & 0 \\
0 & 1
\end{array}\right)+R .
$$

\footnotetext{
${ }^{4}$ For $S L(2, \mathbb{R})$ with a positive level simply substitute $i \alpha$ for $\alpha$ in every equation.
} 
The scalar multiplication $(\cdot)$ is the one in $O(1,1)$, so for example $\underline{v}^{T} \cdot \underline{v} \equiv-v_{1}^{2}+v_{2}^{2}$. One can write the same action as a complete square

$$
\begin{aligned}
S & =S[g]+S^{\prime}[x]+ \\
& +\frac{1}{2 \pi} \int d^{2} z\left[\left(a+\frac{\overline{\mathbf{J}}^{T} \cdot \underline{u}}{2 \underline{u}^{T} M \cdot R \underline{u}}\right) 2 \underline{u}^{T} M \cdot R \underline{u}\left(\bar{a}+\frac{\mathbf{J}^{T} \cdot R \underline{u}}{2 \underline{u}^{T} M \cdot R \underline{u}}\right)-\frac{\left(\overline{\mathbf{J}}^{T} \cdot \underline{u}\right)\left(\mathbf{J}^{T} \cdot R \underline{u}\right)}{2 \underline{u}^{T} M \cdot R \underline{u}}\right]
\end{aligned}
$$

After integrating out the fields $A$ and $\bar{A}$, one gets, to the first order in $\frac{1}{\kappa}$, the action

$$
S=S[g]+S^{\prime}[x]-\frac{1}{4 \pi} \int d^{2} z\left[\frac{\left(\overline{\mathbf{J}}^{T} \cdot \underline{u}\right)\left(\mathbf{J}^{T} \cdot R \underline{u}\right)}{\underline{u}^{T} M \cdot R \underline{u}}\right]
$$

and the dilaton becomes

$$
\Phi=\Phi_{0}-\frac{1}{2} \log \left(\underline{u}^{T} M \cdot R \underline{u}\right) .
$$

The gauge invariance of the action is fixed by setting

$$
g=e^{\frac{1}{2} y \sigma_{3}} g\left(\theta_{i}\right) e^{-\frac{1}{2} y \sigma_{3}} .
$$

The definition of the factor $g\left(\theta_{i}\right)$ depends on the region where $g$ is in the $S L(2)$ group manifold [6, 31]. Defining

$$
W=\operatorname{Tr}\left(\sigma_{3} g \sigma_{3} g^{-1}\right),
$$

$g\left(\theta_{1}\right)$ stands for $e^{i \theta_{1} \sigma_{2}}$ in regions of $S L(2)$ for which $W$ satisfies $|W| \leq 2$. The points of $S L(2)$ for which $W>2$ are divided into 4 regions. There the factor $g\left(\theta_{2}\right)$ represents $\pm e^{ \pm \theta_{2} \sigma_{1}}$. For the 4 regions where $W<-2, g\left(\theta_{3}\right)= \pm i \sigma_{2} e^{ \pm \theta_{3} \sigma_{1}}$. At the point $\theta_{1}=0$, $W=2$. Here two of the regions parameterized by $\theta_{2}$ meet the region parameterized by $\theta_{1}$. Similarly, at $\theta_{1}=\pi$ the other two regions parameterized by $\theta_{2}$ meet the region parameterized by $\theta_{1}$. At $\theta_{1}=\frac{\pi}{2}(W=-2)$ two regions parameterized by $\theta_{3}$ meet the $\theta_{1}$ region and at $\theta_{1}=\frac{3 \pi}{2}$ the other two $\theta_{3}$ regions meet the $\theta_{1}$ region (see figure 3 ). The range of $\theta_{2,3}$ is $0 \leq \theta_{2,3}<\infty$. For the group $S L(2), \theta_{1}$ satisfies $0 \leq \theta_{1} \leq 2 \pi$. For the infinite cover of $S L(2), \theta_{1}$ satisfies $-\infty<\theta_{1}<\infty$. For $P S L(2)$ (or the Poincaré patch), $0<\theta_{1} \leq \pi$.

After plugging (56) into (54), (55) one gets

$$
\begin{gathered}
S=\frac{1}{2 \pi} \int_{\Sigma} \partial x \bar{\partial} x+\frac{\kappa}{2 \pi} \int d^{2} z\left[\partial \theta_{1} \bar{\partial} \theta_{1}-\sin ^{2}\left(\theta_{1}\right) \partial y \bar{\partial} y\right]+ \\
+\frac{1}{\pi} \int d^{2} z \frac{\left(\sqrt{\kappa} \sin ^{2}\left(\theta_{1}\right) \underline{u}_{1} \bar{\partial} y+\underline{u}_{2} \bar{\partial} x\right)\left(\sqrt{\kappa} \sin ^{2}\left(\theta_{1}\right)(R \underline{u})_{1} \partial y-(R \underline{u})_{2} \partial x\right)}{\underline{u}^{T} M \cdot R \underline{u}} \\
\Phi=\Phi_{0}-\frac{1}{2} \log \left(\underline{u}^{T} M \cdot R \underline{u}\right)
\end{gathered}
$$

where $|W| \leq 2$. In the regions where $W>2, \theta_{1}$ in (58), (59) should be replaced by $i \theta_{2}$. In the regions with $W<-2$, substitute $i \theta_{3}$ for $\theta_{1}-\frac{\pi}{2}$.

If we take the vector $\underline{u}^{T}=(1,0)$ then $G_{x, x}$ is constant ${ }^{5}$ and after resealing $x \rightarrow \sqrt{\kappa} x$

\footnotetext{
${ }^{5}$ Actually, $G_{x, x}=$ const iff $(G+B)_{y, x}=0$ and, therefore, in this case the $\frac{S L(2) \times U(1)}{U(1)}$ background can be used in the heterotic string.
} 
the action and the dilaton becomes

$$
\begin{gathered}
S=\frac{1}{2 \pi} \int_{\Sigma} \partial x \bar{\partial} x+\frac{\kappa}{2 \pi} \int d^{2} z\left[\partial \theta_{1} \bar{\partial} \theta_{1}-\sin ^{2}\left(\theta_{1}\right) \partial y \bar{\partial} y\right]- \\
-\frac{1}{\pi} \int d^{2} z \frac{\sqrt{\kappa} \sin ^{2}\left(\theta_{1}\right) \bar{\partial} y\left(\sqrt{\kappa} \sin ^{2}\left(\theta_{1}\right) \cosh (\alpha) \partial y-\sinh (\alpha) \partial x\right)}{1+\cosh (\alpha) \cos \left(2 \theta_{1}\right)}= \\
=\frac{\kappa}{2 \pi} \int d^{2} z\left[\partial \theta_{1} \bar{\partial} \theta_{1}-\frac{\partial y \bar{\partial} y-2 \tanh \left(\frac{\alpha}{2}\right) \bar{\partial} y \partial x}{\cot ^{2}\left(\theta_{1}\right)-\tanh ^{2}\left(\frac{\alpha}{2}\right)}+\partial x \bar{\partial} x\right] \\
\Phi=\tilde{\Phi}_{0}-\frac{1}{2} \log \left(\cos ^{2}\left(\theta_{1}\right)-\tanh ^{2}\left(\frac{\alpha}{2}\right) \sin ^{2}\left(\theta_{1}\right)\right)
\end{gathered}
$$

Again, in regions for which $|W|>2$ make the appropriate replacement for $\theta_{1}$. For large $\kappa$ and for small radius of the circle parameterized by $x$, this action (60) describes a 2-dimensional time-dependent space-time parameterized by $\left(\theta_{i}, y\right)$. The 3-dimensional metric and antisymmetric tensor read from (58) produce a corresponding 2-dimensional structure via the Kaluza-Klein mechanism. The term proportional to $\partial x \bar{\partial} y$ gives rise in two dimensions to a $U(1)$ gauge field whose charge is the momentum as well as the winding along the $x$ circle. The 2-dimensional metric and background gauge field take the form

$$
\begin{aligned}
\frac{1}{\kappa} d s^{2} & =d \theta_{1}^{2}-\frac{\cot ^{2}\left(\theta_{1}\right)}{\left(\cot ^{2}\left(\theta_{1}\right)-\tanh ^{2}\left(\frac{\alpha}{2}\right)\right)^{2}} \partial y \bar{\partial} y \\
A_{y} & =\frac{\sqrt{\kappa} \tanh \left(\frac{\alpha}{2}\right)}{\cot ^{2}\left(\theta_{1}\right)-\tanh ^{2}\left(\frac{\alpha}{2}\right)}
\end{aligned}
$$

In regions for which $|W|>2$ make the appropriate replacement for $\theta_{1} \cdot{ }^{6}$

In the Kruskal coordinates ${ }^{7}$ (for $|W| \leq 2$ )

$$
u=\sin \left(\theta_{1}\right) e^{y}, \quad v=\sin \left(\theta_{1}\right) e^{-y}
$$

the metric, dilaton and gauge field $((62),(61))$ are:

$$
\begin{aligned}
& \frac{1}{\kappa} d s^{2}=\frac{v^{2} d u^{2}+u^{2} d v^{2}}{4 u v}\left(\frac{1}{1-u v}-\frac{1-u v}{\left(1-u v\left[1+\tanh ^{2}\left(\frac{\alpha}{2}\right)\right]\right)^{2}}\right) \\
&+\frac{d u d v}{2}\left(\frac{1}{1-u v}+\frac{1-u v}{\left(1-u v\left[1+\tanh ^{2}\left(\frac{\alpha}{2}\right)\right]\right)^{2}}\right) \\
& A_{u}=\frac{\sqrt{\kappa}}{2} \frac{v \tanh \left(\frac{\alpha}{2}\right)}{1-u v\left(1+\tanh \left(\frac{\alpha}{2}\right)\right)}, \quad A_{v}=-\frac{\sqrt{\kappa}}{2} \frac{1}{1-u v\left(1+\tanh \left(\frac{\alpha}{2}\right)\right)}
\end{aligned}
$$

For the degenerate case $\alpha=0$, the metric, dilaton and background gauge field can be written as follows

$$
\begin{aligned}
d s^{2} & =-\kappa \frac{d u d v}{1-u v} \\
\phi & =-\frac{1}{4} \log (1-u v)^{2}
\end{aligned}
$$

\footnotetext{
${ }^{6}$ The charged 2-d black hole $\frac{S L(2)_{k>0} \times U(1)}{U(1)_{\text {space }}-\text { like }}$ is the same as (60), (61), (62) with $\alpha \rightarrow i \alpha, x \rightarrow i x$ and $\kappa \rightarrow-\kappa$.

${ }^{7}$ The coordinates $u$ and $v$ cover all $\frac{P S L(2)}{U(1)}$, and only half of $\frac{S L(2)}{U(1)}$ (or the Poincaré patch of the universal cover).
} 


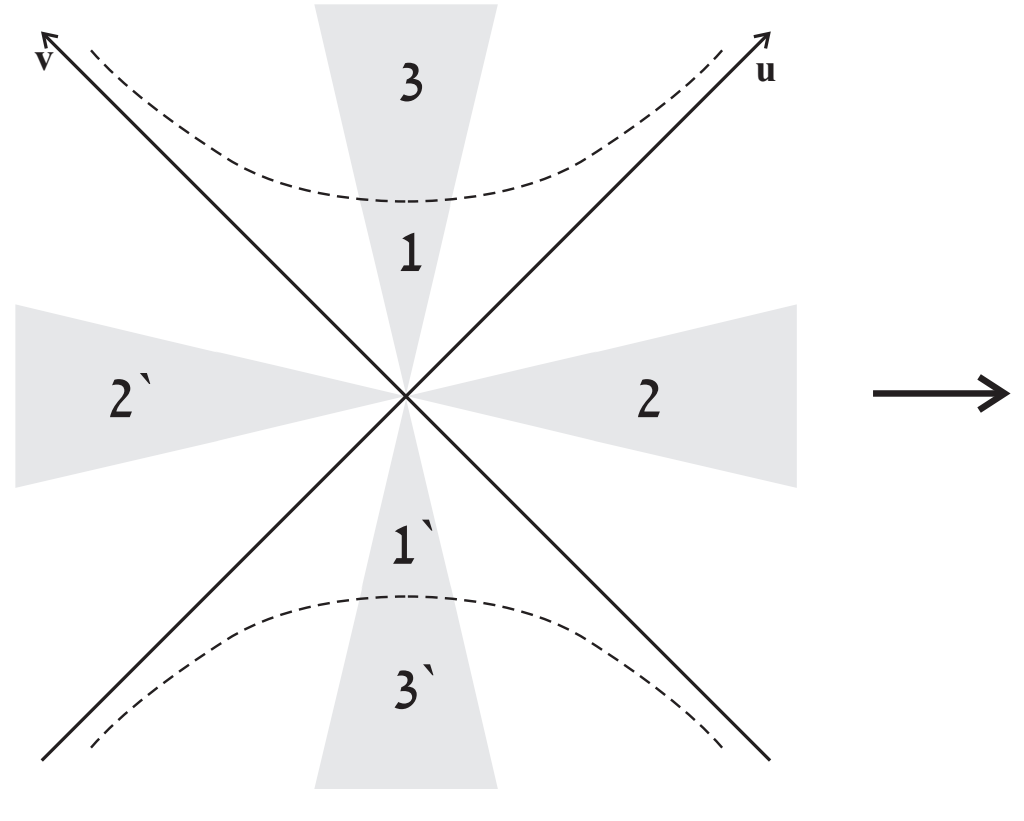

a

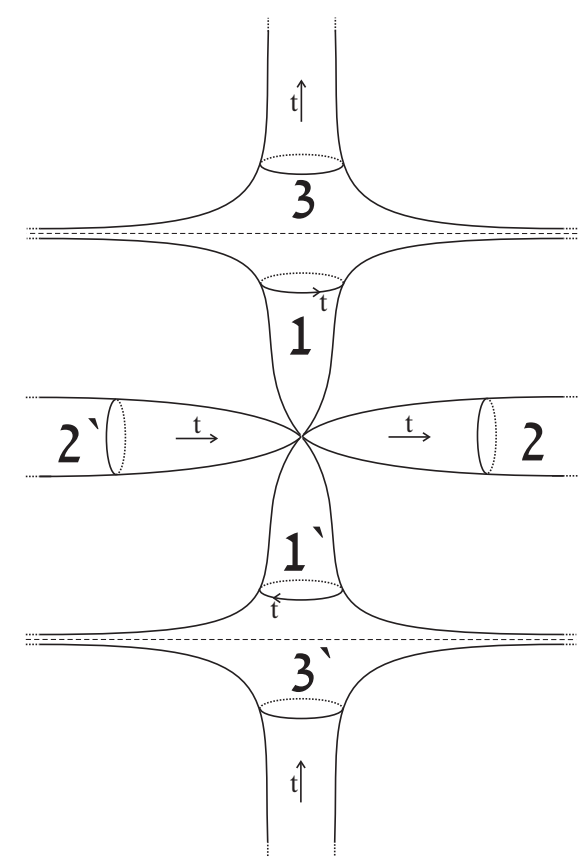

b

Figure 3: a: The two dimensional cosmology where the fundamental domains are marked; the dashed lines are curvature singularities. b: The space after the identification.

This is the two dimensional Lorentzian black hole background with a negative level.

So far we have constructed $\frac{S L(2) \times U(1)}{\mathbb{R}}$. To get $\frac{S L(2) \times U(1)}{U(1) \times Z}$ we further identify

$$
g \sim e^{\pi \lambda \sigma_{3}} g e^{-\pi \lambda \sigma_{3}} \Longleftrightarrow y \sim y+2 \pi \lambda
$$

The identification in $S L(2)$ is the one which leads to the BTZ black hole background (with a negative level), so our space is $\frac{B T Z_{k<0} \times U(1)}{U(1)}$.

In the degenerate case $\alpha=0,(68)$ becomes $(u, v) \sim\left(u e^{-\lambda}, v e^{\lambda}\right)$. This space was recently studied in [16] and is plotted in figure 3. Regions 2,2',3,3' are time-dependent, and approach flat space at early or late times. Regions 1,1' are static and have closed time-like curves. Regions $1,1^{\prime}, 2,2^{\prime}$ meet at an orbifold singularity which is locally an $R^{1,1}$ modded by boost singularity. Regions 1 and 3 meet at a curvature singularity which coincides with an orbifold singularity, and the same for regions 1' and 3'. The lines $u=0$ and $v=0$ in figure $3 \mathbf{a}$ lead to a non-Hausdorf structure which is not indicated in figure $3 \mathrm{~b}$.

When we turn on $\alpha \neq 0$, the curvature singularities move into the compact static regions (1 and $\left.1^{\prime}\right) .{ }^{8}$ In $\frac{S L(2) \times U(1)}{U(1) \times Z}$ it leaves an orbifold singularity behind (see figure 4). It is an orbifold singularity since at $\theta_{1}=\frac{\pi}{2}$ one can do a gauge transformation together with the orbifold identification (68) such that it acts only in the $U(1)$ part and, therefore, it becomes a fixed point after KK reduction. If we where considering the space with no orbifold identification or KK reduction, the surface $\theta_{1}=\frac{\pi}{2}$ would have been a horizon instead of being a singularity.

\footnotetext{
${ }^{8}$ In the case of a positive level $k>0$ (when the resulting quotient is a charged black hole), the curvature singularity is moved into what is now a static, non-compact region.
} 

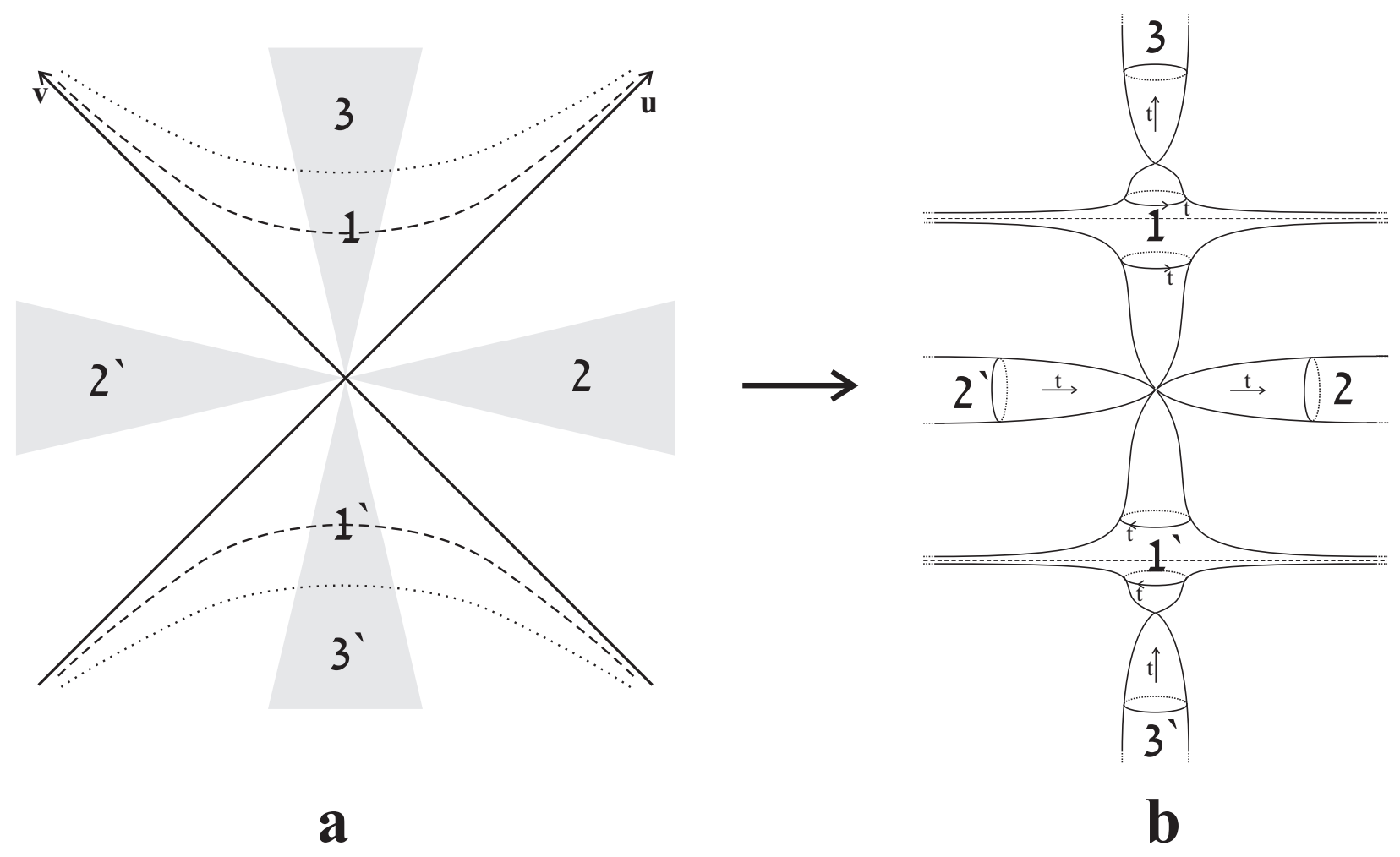

Figure 4: a: The 2-dimensional cosmology with a background gauge field $(\alpha \neq 0)$; the dashed lines are curvature singularities and the doted lines are horizons. b: The space after the identification; the dashed lines are time-like curvature singularities - domain walls - in the static compact regions. The horizons in a turned into big bang/crunch orbifold singularities, connecting the various regions.

To summarize, we considered a two dimensional cosmology with a positive cosmological constant, in the presence of an Abelian gauge field. As in [7], we find that turning on an Abelian gauge field in the background studied in [16], changes the structure of the singularities. The singularities split into a curvature singularity and orbifold singularities. The curvature singularities become time-like, and are located inside the compact static regions. On the other hand, the orbifold $R^{1,1}$ Boost-like singularities are big bang and crunch singularities of the expending and contracting universes, respectively. Unlike ref. [7], where the curvature singularities can be removed completely by tuning the gauge field such that they are pushed towards the boundaries of the whiskers in figure 1, here the singularities cannot be removed. They are "stuck" inside the compact static regions.

The fact that this background is an $\frac{S L(2) \times U(1)}{U(1) \times Z}$ CFT sigma-model allows to extract some exact results. For instance, the Bogolubov coefficients computed in [16] eq. (4.32) apply to our $\alpha$-family as well; only the dispersion relation is modified as a function of the $\alpha$-dependent gauge condition. As a consequence, particle creation remains for a generic gauge field (eq. (4.37) in [16] is valid here as well).

Acknowledgements: We are grateful to S. Elitzur and D. Kutasov for collaborations. This work is supported in part by BSF - American-Israel Bi-National Science Foundation, the Israel Academy of Sciences and Humanities - Centers of Excellence Program, the German-Israel Bi-National Science Foundation, the European RTN network HPRN-CT- 
2000-00122, and the Horwitz foundation (AS).

\section{References}

[1] For example: W. Israel, "Thermo Field Dynamics Of Black Holes," Phys. Lett. A 57, 107 (1976).

[2] W. Fischler and L. Susskind, "Holography and cosmology," arXiv:hep-th/9806039. R. Bousso, "A Covariant Entropy Conjecture," JHEP 9907, 004 (1999) [arXiv:hepth/9905177].

[3] J. M. Maldacena and A. Strominger, "Statistical entropy of de Sitter space," JHEP 9802, 014 (1998) [arXiv:gr-qc/9801096]. S. Hawking, J. M. Maldacena and A. Strominger, "DeSitter entropy, quantum entanglement and AdS/CFT," JHEP 0105, 001 (2001) [arXiv:hep-th/0002145]. T. Banks, "Cosmological Breaking Of Supersymmetry?," Int. J. Mod. Phys. A 16, 910 (2001).

[4] see for example: S. W. Hawking, "The Chronology protection conjecture," Phys. Rev. D 46, 603 (1992).

[5] W. A. Hiscock and D. A. Konkowski, "Quantum Vacuum Energy In Taub - Nut (Newman-Unti-Tamburino) Type Cosmologies," Phys. Rev. D 26, 1225 (1982).

[6] S. Elitzur, A. Giveon, D. Kutasov and E. Rabinovici, "From big bang to big crunch and beyond," JHEP 0206, 017 (2002) [arXiv:hep-th/0204189].

[7] S. Elitzur, A. Giveon and E. Rabinovici, "Removing singularities," JHEP 0301, 017 (2003) [arXiv:hep-th/0212242].

[8] P. Horava, "Some exact solutions of string theory in four-dimensions and fivedimensions," Phys. Lett. B 278, 101 (1992) [arXiv:hep-th/9110067]. C. Kounnas and D. Lust, "Cosmological string backgrounds from gauged WZW models," Phys. Lett. B 289, 56 (1992) [arXiv:hep-th/9205046]. A. A. Tseytlin, "Exact string solutions and duality," arXiv:hep-th/9407099. J. Simon, "The geometry of null rotation identifications," arXiv:hep-th/0203201. A. J. Tolley and N. Turok, "Quantum fields in a big crunch / big bang spacetime," arXiv:hep-th/0204091.

[9] N. A. Nekrasov, "Milne universe, tachyons, and quantum group," arXiv:hepth/0203112.

[10] L. Cornalba and M. S. Costa, "A New Cosmological Scenario in String Theory," Phys. Rev. D 66, 066001 (2002) [arXiv:hep-th/0203031].

[11] A. Giveon and A. Pasquinucci, "On cosmological string backgrounds with toroidal isometries," Phys. Lett. B 294, 162 (1992) [arXiv:hep-th/9208076].

[12] I. Bars and K. Sfetsos, " $S L(2, \mathbb{R}) \times S U(2) / \mathbb{R}^{2}$ string model in curved space-time and exact conformal results," Phys. Lett. B 301, 183 (1993) [arXiv:hep-th/9208001].

[13] C. R. Nappi and E. Witten, "A Closed, expanding universe in string theory," Phys. Lett. B 293, 309 (1992) [arXiv:hep-th/9206078].

[14] H. Liu, G. Moore and N. Seiberg, "Strings in a time-dependent orbifold," JHEP 0206, 045 (2002) [arXiv:hep-th/0204168]. 
[15] H. Liu, G. Moore and N. Seiberg, "Strings in time-dependent orbifolds," JHEP 0210, 031 (2002) [arXiv:hep-th/0206182].

[16] B. Craps, D. Kutasov and G. Rajesh, "String propagation in the presence of cosmological singularities," JHEP 0206, 053 (2002) [arXiv:hep-th/0205101].

[17] for example see: O. Aharony, M. Fabinger, G. T. Horowitz and E. Silverstein, "Clean time-dependent string backgrounds from bubble baths," JHEP 0207, 007 (2002) [arXiv:hep-th/0204158].

[18] see for example: A. Sen, "Time evolution in open string theory," JHEP 0210, 003 (2002) [arXiv:hep-th/0207105].

[19] A. Lawrence, "On the instability of 3D null singularities," JHEP 0211, 019 (2002) [arXiv:hep-th/0205288].

[20] J. M. Maldacena, "Eternal black holes in Anti-de-Sitter," arXiv:hep-th/0106112.

[21] S. W. Hawking and D. N. Page, "Thermodynamics Of Black Holes In Anti-De Sitter Space," Commun. Math. Phys. 87, 577 (1983). E. Witten, "Anti-de Sitter space, thermal phase transition, and confinement in gauge theories," Adv. Theor. Math. Phys. 2, 505 (1998) [arXiv:hep-th/9803131].

[22] A. Giveon and A. D. Shapere, "Gauge symmetries of the N=2 string," Nucl. Phys. B 386, 43 (1992) [arXiv:hep-th/9203008]. G. W. Moore, "Finite In All Directions," arXiv:hep-th/9305139. J. Khoury, B. A. Ovrut, P. J. Steinhardt and N. Turok, "Density perturbations in the ekpyrotic scenario," Phys. Rev. D 66, 046005 (2002) [arXiv:hep-th/0109050]. V. Balasubramanian, S. F. Hassan, E. Keski-Vakkuri and A. Naqvi, "A space-time orbifold: A toy model for a cosmological singularity," Phys. Rev. D 67, 026003 (2003) [arXiv:hep-th/0202187]. E. J. Martinec and W. McElgin, "Exciting AdS orbifolds," arXiv:hep-th/0206175. M. Fabinger and J. McGreevy, "On smooth time-dependent orbifolds and null singularities," arXiv:hep-th/0206196. J. Simon, "Null orbifolds in AdS, time dependence and holography," JHEP 0210, 036 (2002) [arXiv:hep-th/0208165].

[23] J. Figueroa-O'Farrill and J. Simon, "Supersymmetric Kaluza-Klein reductions of M2 and M5 branes," arXiv:hep-th/0208107.

[24] K. Bardakci and M. B. Halpern, "New Dual Quark Models," Phys. Rev. D 3, 2493 (1971). P. Goddard, A. Kent and D. I. Olive, "Virasoro Algebras And Coset Space Models," Phys. Lett. B 152, 88 (1985).

[25] A. B. Zamolodchikov, "'Irreversibility' Of The Flux Of The Renormalization Group In A 2-D Field Theory," JETP Lett. 43, 730 (1986) [Pisma Zh. Eksp. Teor. Fiz. 43, 565 (1986)].

[26] K. Bardakci, E. Rabinovici and B. Saering, "String Models With $C<1$ Components," Nucl. Phys. B 299, 151 (1988). D. Altschuler, K. Bardakci and E. Rabinovici, "A Construction Of The $C<1$ Modular Invariant Partition Functions," Commun. Math. Phys. 118, 241 (1988). W. Nahm, "Gauging Symmetries Of TwoDimensional Conformally Invariant Models," UCD-88-02. D. Karabali, Q. H. Park, H. J. Schnitzer and Z. Yang, "A Gko Construction Based On A Path Integral Formulation Of Gauged Wess-Zumino-Witten Actions," Phys. Lett. B 216, 307 (1989). 
K. Gawedzki and A. Kupiainen, "Coset Construction From Functional Integrals," Nucl. Phys. B 320, 625 (1989).

[27] K. Bardacki, M. J. Crescimanno and E. Rabinovici, "Parafermions From Coset Models," Nucl. Phys. B 344, 344 (1990). E. Witten, "On string theory and black holes," Phys. Rev. D 44, 314 (1991).

[28] R. Dijkgraaf, H. Verlinde and E. Verlinde, "String propagation in a black hole geometry," Nucl. Phys. B 371, 269 (1992). A. A. Tseytlin, "Conformal sigma models corresponding to gauged Wess-Zumino-Witten theories," Nucl. Phys. B 411, 509 (1994) [arXiv:hep-th/9302083]. H. J. de Vega, A. L. Larsen and N. Sanchez, "Nonsingular string-cosmologies from exact conformal field theories," Nucl. Phys. Proc. Suppl. 102, 201 (2001) [arXiv:hep-th/0110262].

[29] M. Gasperini and G. Veneziano, "Pre - big bang in string cosmology," Astropart. Phys. 1, 317 (1993) [arXiv:hep-th/9211021]. R. Brustein and G. Veneziano, "The Graceful exit problem in string cosmology," Phys. Lett. B 329, 429 (1994) [arXiv:hep-th/9403060]. G. Veneziano, "String cosmology: The pre-big bang scenario," arXiv:hep-th/0002094. J. Khoury, B. A. Ovrut, N. Seiberg, P. J. Steinhardt and N. Turok, "From big crunch to big bang," arXiv:hep-th/0108187.

[30] R. Dijkgraaf, H. Verlinde and E. Verlinde, "String propagation in a black hole geometry," Nucl. Phys. B 371, 269 (1992).

[31] N.J. Vilenkin, "Special Functions and the Theory of Group Representations" AMS, 1968; N.J. Vilenkin, A.U. Klimyk "Representation of Lie Groups and Special Function" Kluwer Academic Publishers, 1991.

[32] P. Kraus, H. Ooguri and S. Shenker, "Inside the horizon with AdS/CFT," arXiv:hepth/0212277.

[33] J. M. Maldacena, "The large N limit of superconformal field theories and supergravity," Adv. Theor. Math. Phys. 2, 231 (1998) [Int. J. Theor. Phys. 38, 1113 (1999)] [arXiv:hep-th/9711200].

[34] G. T. Horowitz and J. Polchinski, "Instability of spacelike and null orbifold singularities," Phys. Rev. D 66, 103512 (2002) [arXiv:hep-th/0206228].

[35] M. Gasperini, J. Maharana and G. Veneziano, "Boosting away singularities from conformal string backgrounds," Phys. Lett. B 296, 51 (1992) [arXiv:hep-th/9209052].

[36] A. Giveon, M. Porrati and E. Rabinovici, "Target space duality in string theory," Phys. Rept. 244, 77 (1994) [arXiv:hep-th/9401139].

[37] A. Giveon and M. Rocek, "Generalized duality in curved string backgrounds," Nucl. Phys. B 380 (1992) 128 [arXiv:hep-th/9112070]. 